\title{
Effect of Excess Calorie Consumption on Depression of Young and Aged \\ Human Males: Impact of Physical Activity
}

\author{
Aindrila Das ${ }^{1,2}$, Samir K Ghosh ${ }^{1}$, Goutam Paul ${ }^{2}$ and Mrinal K Poddar ${ }^{1,3 *}$ \\ ${ }^{1}$ Department of Biochemistry, University of Calcutta, Kolkata, India \\ ${ }^{2}$ Department of Physiology, University of Kalyani, Kalyani, Nadia, West Bengal, India \\ ${ }^{3}$ Department of Pharmaceutical Technology, Jadavpur University, India
}

*Corresponding Author: Mrinal K Poddar, Department of Biochemistry, University of Calcutta, Kolkata, India and Department of Pharmaceutical Technology, Jadavpur University, India. E-mail: mrinalkp@yahoo.com

Received: June 24, 2019; Published: July 11, 2019

DOI: $10.31080 /$ ASNH.2019.03.0364

\begin{abstract}
Depression is less prevalent among older adults than among younger adults but can have serious consequences. Overeating, inactivity, and obesity have emerged as new challenges in manifestation of depressive symptoms. To study the influence of excess calorie intake and physical activity level on depression of a randomized human male population in urbanized areas of West Bengal, India, the present cohort study was carried out with 357 young (30.13 $\pm 0.24 \mathrm{yr}$ ) and 287 aged (58.62 $\pm 0.29 \mathrm{yr}$ ) subjects. Participants' socioeconomic status (according to Kuppuswamy's socio-economic scale), health status (as per health assessment questionnaire; HAQ), calorie and micronutrient consumption (considering Diet Survey - Three 24h Recall), depression level (using CES-D scale) and physical activity level (PAL) (as per questionnaire) were assessed. No significant differences in depression level was found between At Par (AP) and Excess Calorie (CE) consuming subjects of young and aged groups. However the percent population of depressed aged subjects consuming CE diet was significantly higher compared to young of the same calorie consuming group. High PAL effectively masked the occurrence of depression in the CE diet-consuming aged subjects compared to CE diet consuming young with high PAL. The present study suggests that CE diet in the aged, unlike AP calorie diet, increases the percent population of depressed subjects. Young subjects do not exhibit any significant difference in depressive outcome when on CE diet compared to their AP calorie consuming counterpart. Further, High PAL masks the excess calorie- induced depressive symptoms in aged subjects.
\end{abstract}

Keywords: Physical Activity Level; Excess Calorie; Aging; Depression

\section{Introduction}

WE live in an aging society. In the next 30 years, there will be a rise in elderly population of up to $300 \%$ in Asia and Latin America $[1,2]$. Aging refers to a deterioration of multidimensional processes in humans, at the level of Physiological, Biochemical and even social [3]. Increased longevity is associated with an increase in multiple chronic conditions that sometimes translate into functional disability and need for assistance [4]. The extra years can be marked by declining health, reduced mobility, depression, isolation, and loneliness [5] Health and functioning of older adults are influenced by many factors other than biological senescence. Demographic, social, and environmental factors, including physical activity and dietary habits, play a major role. Fortunately, many of these societal factors are amenable to public health interventions and programs [6]. Among the most important self-care behaviors are those that involve physical activity and diet. In view of the rapid growth of aged population continued good health of the elderly population is a major challenge to public health [7].
In many past studies the success of the efforts at health promotion has been measured in terms of lower mortality rates or reduced disease risk. There is increasing concern that these classic medical endpoints (mortality and morbidity) may not adequately represent functional impairments and disabilities during the later years of life. Increasingly, health is viewed as not only the absence of infirmity and disease but also as a state of physical, mental, and social well-being $[8,9]$. Much progress has been made in establishing a broader conceptual framework of health status for older adults [10]. Health-related quality of life (HRQL), a relatively new concept, expands the morbidity- and mortality-based definition of health to include a personal sense of physical and mental health, social functioning, and emotional well-being. Other and more global measures of quality of life are even more inclusive, taking overall life satisfaction and happiness into account [11,12]. Quality-of-life measures permit researchers to compare the status of different groups over time and assess the effectiveness of public health interventions and programs [4]. 
Of many factors, sleep disturbance, consisting of inadequate sleep and sleep deprivation are common among the aged, which often leads to manic and depressive episodes [13]. Depression is more typically thought of as strictly biochemical-based or emotionally-rooted. On the contrary, nutrition can play a key role in the onset as well as severity and duration of depression. Many of the easily noticeable food patterns that precede depression are the same as those that occur during depression. These may include poor appetite, skipping meals, and a dominant desire for sweet foods [14]. It has been reported that young adults (18-29yrs) are more vulnerable to depression and the number has been shown to be positively correlated to the level of education [15]. It has also been shown that a high proportion of community-dwelling elderly persons suffer from significant symptoms of depression [16]. Other than sleep, diet and exercise are important influences on development, progression and treatment of depression [17]. The work of Anders Abildgaard., et al. [18] have suggested from their study on rats with a genetic model of depression that metabolic stress induced by high calorie intake/high fat diet developed aberrant behaviors related to depression. Depression maintains a correlation with physical activity levels. The work of Camacho., et al. 1991 have shown that adjustments for physical health, socioeconomic status, life events, social supports, and other health habits do not affect the association appreciably [19]. A significant number of studies examining the relation between calorie intake and obesity [20] make it logical to view calorie (excess) consumption as a predictor of obesity. However the propensity to obesity (higher BMI) among individuals with depression is also sometimes observed in patients who live in apparently-supportive environments and who have not been treated with psychotropic drugs that are recognized as promoting obesity [21]. Bidirectional associations have been reported from a meta-analysis of longitudinal studies [21] where it has been found that obesity increased the risk of depression by $55 \%$ and depression increased the risk of becoming obese by $58 \%$.

Nutrition is an important determinant of health in elderly [22]. Malnutrition is both a cause and a consequence of ill health [23]. It can be of various types: undernutrition, overnutrition, or specific nutrient-related deficiencies. Malnutrition in older individuals is regularly underdiagnosed [24].

The data on the efficacy of exercise as an intervention for Major Depressive Disorder (MDD) and bipolar disorder have yielded effect sizes comparable to medications [25]. Preliminary research also reports the benefits of exercise augmentation strategies added to cognitive-behavioral therapy for anxiety disorders or treatment as usual for depression [26].

Very few studies have explored the interrelationships among dietary measures, physical activity variables, and quality-of-life in older adults or the nature of the intervening variables. The aim of the present study is to assess the mental health (depression level) of an aged population sampled from an urban and suburban areas of the state of West Bengal, who belong to lower- middle and upper- lower $[27,28]$ classes and happened to be exposed to variable micronutrient deficiency. The study further aims to examine the effects of intervention of excess calorie consumption and physical activity on depression. The novelty of the study lies in the method of random sampling of subjects of a definite socioeconomic class from within a population consuming differential calorie, having different PAL and includes both young and aged for comparison. In addition, the entire study population is exposed to micronutrient deficiency, the impact of which on mental health is undetermined.

\section{Materials and Methods}

\section{Subjects}

In the present investigation human male subjects were selected from the district of Kolkata and suburbs in the state of West Bengal, India. The work was initiated with a total randomized sample population of 1032 human male subjects belonging to either Lower-middle or Upper-lower class, as assessed according to Kuppuswamy's socio-economic scale [27,28].

The diagrammatic representation (Figure 1) explains the flow of participants during the work. The present study has been carried out with only at par (AP) and excess calorie (CE) consuming above mentioned subjects under prevailing micronutrient deficiency as evaluated using dietary survey [29-31]. Accordingly the working sample size has been reduced to $644(\mathrm{~N})$ from 1032 out of which 357 were young (20 -35 years) and 287 were aged (55 -70 years). The individuals considered in this study were free from any addiction (self- reported). The health status assessed by the Health Assessment Questionnaire (HAQ) [32] of the subjects considered in the study did not reflect any disability or morbidity. Subjects were selected from groups of morning walker, free health checkup camps, construction site, market place, academic institution etc.

\section{General procedure}

The subjects were interviewed in a very planned manner. Consent were taken either earlier from the subjects (as for morning walkers) with subsequent fixing of date and time of interview or similar arrangements were made with institutions, local clubs providing health check-up facilities to local low-income people, or the head of a community in the village. In all instances the subjects were briefed about what they were required to do and how. Rejection rate to comply with the interview process varied between 5 to $25 \%$ depending on the subjects' pre - occupation.

\section{Assessment}

\section{Calorie consumption}

The calorie consumption by individual subject was assessed from the estimation of food and nutrient intake, i.e. Diet Survey. The 
Diet Survey was carried out following three $24 \mathrm{~h}$ Recall Method as described by Gibson and Ferguson [29,31], and validated by Yunsheng., et al. [30].

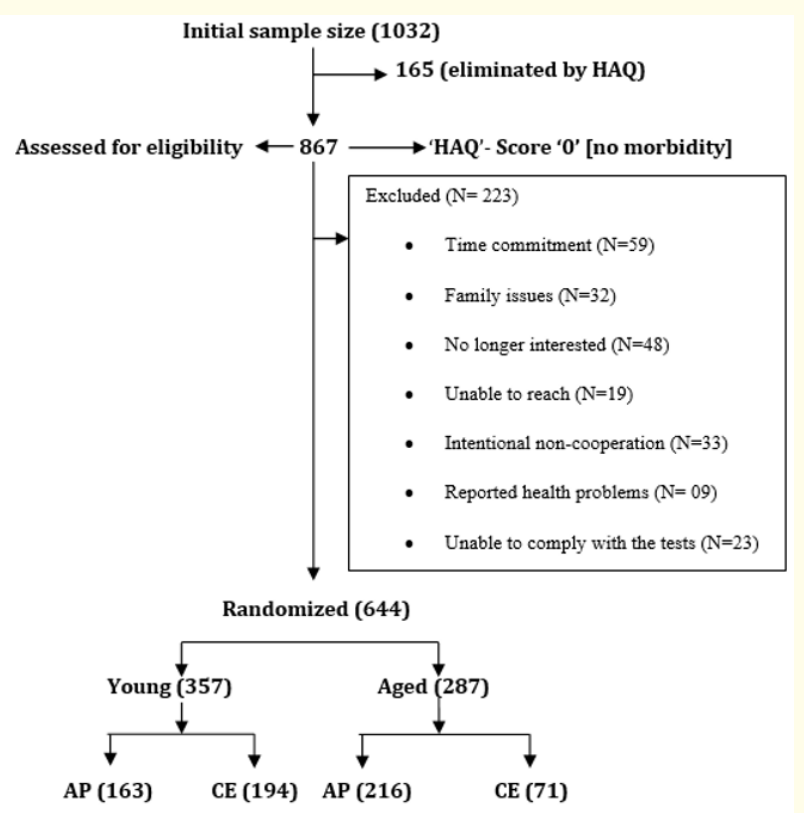

Figure 1: Flow of subjects through the study. Number in the parenthesis indicates sample size in respective category.

\section{Depression status}

The depressive status of the experimental human subjects was measured by using The Center for Epidemiological Studies-Depression Scale (CES-D) as described by Radloff [33]. The scale, in the form of a questionnaire was asked among the studied subjects and the same were classified as non-depressed and depressed individuals from the score of the CES-D Scale. The subjects were given a set of behavioral queries that they were required to grade. Scoring was done from the grades and the scale assigned those subjects as non-depressed whose score was between the range of 0 to 15 and those score equal to or greater than 16 , was considered as depressed individuals [33]. The CES-D is intended to be completed independently by the subject.

\section{Physical activity level (PAL)}

Physical activity level (PAL) was assessed by following the standard questionnaire where the subjects' self-reported average daily activities in $24 \mathrm{hr}$ were recorded and then the average energy expenditure of each individual in performing the different activities for 24 hour was calculated following the method as described by Bharathi., et al [34]. The method followed was by factorial estimation of total energy expenditure and physical activity level [35].

\section{Statistical analysis}

Demographic characteristics, calorie intake and BMI were expressed as mean \pm SEM. The numerical differences with respect to BMI and calorie intake were calculated using student's t-test [36]. Significance of difference of depression between relevant groups with respect to age, calorie intake and physical activity level (PAL) were calculated using one-way analyses of variance (ANOVA) followed by post-hoc Tukey's HSD test [37]. Qualitative differences of depression status with respect to age, calorie intake and PAL were calculated by using binomial logistic regression analysis [38].

Binomial logistic regression analysis was used to evaluate the influence of age, calorie intake and PAL on the results of depression status. Test results were used as response variables whereas age, calorie intake and PAL were used as predictors in the logistic regression models.

Linear correlation coefficients were calculated between age/ calorie consumption/physical activity level and depression. In all cases differences were considered significant if $\mathrm{p}<0.05$. All statistical analyses were done using SPSS (SPSS Version 20.0, SPSS Inc., Chicago, IL).

\section{Results}

Figure 2 describes the demographic characteristics and calorie intake of young and aged human male subjects who participated in the present study. The subjects in each age group were categorized as At Par (AP) and Excess Calorie (CE) based on their calorie consumption. Percent population of each calorie category is given in parenthesis. The mean BMI of young and aged subjects on CE diet is significantly $(p<0.001)$ higher than that of the corresponding AP calorie consuming subjects. Mean calorie consumption of AP subjects significantly $(\mathrm{p}<0.001)$ differs from the mean calorie value consumed by the subjects in the CE category and it holds for both young and aged subjects.

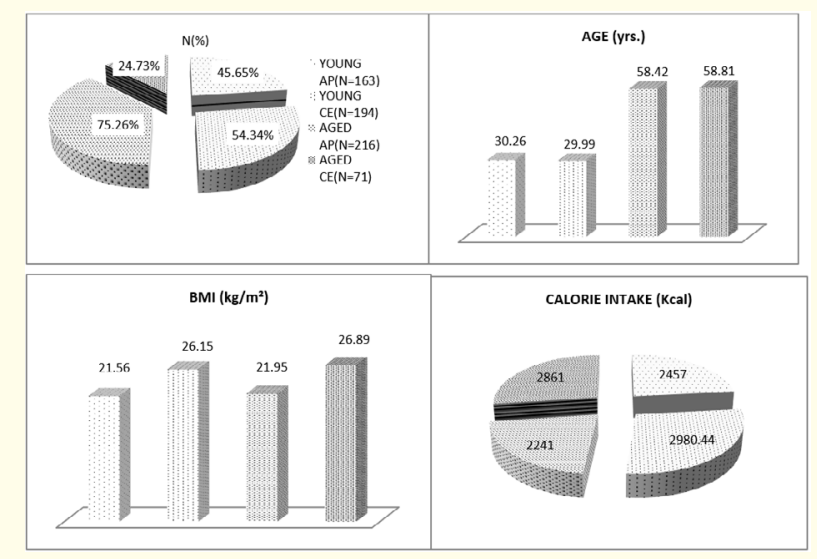

Figure 2: Demographic characteristics and Calorie intake of the participants. 
Table 1 shows the average amount of micronutrient available in the diet consumed by the same study population as shown in Figure 2. Dietary micronutrient analysis reflects a prevalence of deficiency of certain micronutrient and vitamin such as $\mathrm{Ca}^{2+}, \mathrm{Zn}^{2+}$, riboflavin $\left(\mathrm{B}_{2}\right)$, pyridoxine $\left(\mathrm{B}_{6}\right)$ and cobalamin $\left(\mathrm{B}_{12}\right)(\mathrm{p}<0.05)$ in the whole study population compared to their recommended dietary allowance (RDA) [39].

\begin{tabular}{|l|c|c|}
\hline Micronutrients & Dietary intake of micronutrients & $\begin{array}{c}\text { Dietary Reference Intakes (DRIs): } \\
\text { Estimated Average Requirements* }\end{array}$ \\
\hline Calcium $(\mathrm{mg} / \mathrm{d})$ & $478.03 \pm 21.10$ & 800.0 \\
\hline Iron $(\mathrm{mg} / \mathrm{g})$ & $24.35 \pm 1.30$ & 6.0 \\
\hline Zinc $(\mathrm{mg} / \mathrm{d})$ & $6.54 \pm 0.46$ & 9.4 \\
\hline Magnesium $(\mathrm{mg} / \mathrm{d})$ & $446.50 \pm 29.88$ & 350.0 \\
\hline Vitamin $C(\mathrm{mg} / \mathrm{d})$ & $93.90 \pm 5.65$ & 75.0 \\
\hline Thiamine $\left(\mathrm{B}_{1}\right)(\mathrm{mg} / \mathrm{d})$ & $1.53 \pm 0.09$ & 1.0 \\
\hline Riboflavin $\left(\mathrm{B}_{2}\right)(\mathrm{mg} / \mathrm{d})$ & $0.79 \pm 0.03$ & 1.1 \\
\hline Niacin $\left(\mathrm{B}_{3}\right)(\mathrm{mg} / \mathrm{d})$ & $21.84 \pm 1.45$ & 12.0 \\
\hline Pyridoxine $\left(\mathrm{B}_{6}\right)(\mathrm{mg} / \mathrm{d})$ & - & 1.4 \\
\hline Cobalamin $\left(\mathrm{B}_{12}\right)(\mu \mathrm{dg} / \mathrm{d})$ & - & 2.0 \\
\hline
\end{tabular}

Table 1: Micronutrient consumption status of study population

Results expressed as mean \pm SEM of observed number of subjects in the present study.

*Sources: Dietary Reference Intakes for Calcium, Phosphorous, Magnesium, Vitamin D, and Fluoride (1997); Dietary Reference Intakes for Thiamin, Riboflavin, Niacin, Vitamin B , Folate, Vitamin B , Pantothenic Acid, Biotin, and Choline (1998); Dietary Reference Intakes for Vitamin C, Vitamin E, Selenium, and Carotenoids (2000); Dietary Reference Intakes for Vitamin A, Vitamin K, Arsenic, Boron, Chromium, Copper, Iodine, Iron, Manganese, Molybdenum, Nickel, Silicon, Vanadium, and Zinc (2001); Dietary Reference Intakes for Energy, Carbohydrate, Fiber, Fat, Fatty Acids, Cholesterol, Protein, and Amino Acids (2002/2005); and Dietary Reference Intakes for Calcium and Vitamin D (2011). These reports may be accessed via www.nap.edu.

Figure 3 shows that among the young $(n=163)$ with AP calorie diet $71.78 \%$ were not-depressed and $28.22 \%$ were depressed. In the same age group but on CE diet, $64.95 \%$ out of 194 subjects were not-depressed and 35.05\% were depressed. The results (Figure 3) also revealed that no significant difference ( $>>0.05)$ were observed in term of change in percent population of depressed and not-depressed individuals when compared between young AP and young CE and aged AP and aged CE. Of all the groups studied, only significant $(\mathrm{p}<0.001)$ difference in the percent population change of depressed (48.67\%) and not-depressed (26.27\%) subjects were seen between young CE and aged CE.

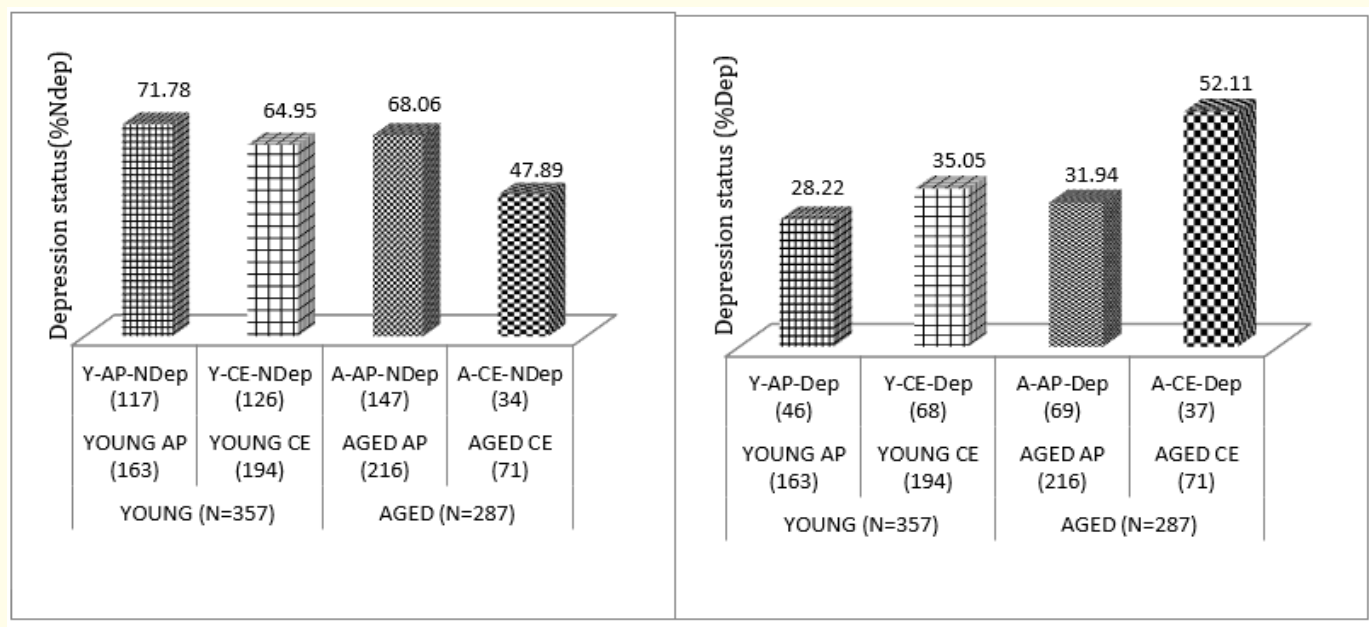

Number within parenthesis represents number of subjects in respective groups. Y represents Young; A represents Aged; AP indicates At Par, CE indicates Calorie Excess (> 30\% of calorie requirement); NDep represents not-depressed, Dep represents depressed.

Figure 3: Effect of Excess Calorie consumption on Depression status. 
It is evident from Figure 4(a) that out of 101 young subjects having AP calorie diet with high PAL 92.08\% were not-depressed and $7.92 \%$ were depressed in comparison to $38.71 \%$ and $61.29 \%$ not-depressed and depressed respectively out of 62 subjects in the same age and calorie group but with low PAL. Thus, the results reveal that the change in per cent population of not-depressed young subjects having AP calorie diet with low PAL was significantly lower (57.96\%; $\mathrm{p}<0.001)$ in comparison to that observed in the young AP calorie group but with high PAL; while that of young depressed subjects having AP calorie diet with low PAL was significantly higher $(673.86 \%$; $p<0.001)$ compared to young AP subjects with high PAL. Results of Figure-4(b) show that out of 115 aged subjects in the AP calorie group with high PAL, 93.91\% were not-depressed and $6.09 \%$ were depressed but with low PAL out of 101 subjects, $36.63 \%$ were not-depressed and $63.37 \%$ were depressed in the same age and calorie group. Thus among aged having AP calorie diet and with low PAL a significant decrease (60.99\%; $\mathrm{p}<0.001$ ) in not-depressed population and a significant increase $(940.56 \%$, $\mathrm{p}<0.001$ ) in depressed population were observed compared to their high PAL counterpart.

When depression status of aged subjects on AP calorie diet with high PAL was compared to their young counterpart no significant difference ( $p>0.05$ ) in percent population either of depressed or of not-depressed subjects was observed. Also, when young subjects on AP calorie diet but with low PAL were compared to their corresponding aged populations, aged subjects on AP calorie diet with low PAL showed no significant change ( $p>0.05$ ) in percent population either of depressed or of not-depressed subjects. Again, when young and aged subjects on AP calorie diet but with low PAL were compared to their corresponding aged and young populations with high PAL, young subjects on AP calorie diet with low PAL showed significantly higher $(906.40 \% \mathrm{p}<0.001)$ depressed population compared to the aged subjects on AP calorie diet with high PAL; while aged subjects on AP calorie diet with low PAL exhibited significantly higher $(700.13 \% ; \mathrm{p}<0.001)$ depressed population compared to young subjects on AP calorie diet but with high PAL.

When both young and aged subjects were on CE diet and with low PAL [Figure 4 (a) and (b)] they both exhibited greater percentage of depressed population (young $=64.20 \%$, aged $=70.83 \%$ ). Figure 4 (a) and (b) further reveals that excess calorie consum- ing young subjects with low PAL showed significantly higher (392.33\%; $\mathrm{p}<0.001$ ) depressed population compared to aged high PAL subjects in the same calorie group. Results also revealed [Figure 4(a) and (b)] that excess calorie consuming aged subjects with low PAL showed a significant increase $(400.21 \%$; $<<0.001)$ in depressed population when compared with young subjects in same calorie group but with high PAL.

Figure 4(a) also shows that out of 113 subjects in the young CE diet consuming group with high PAL $85.84 \%$ were not-depressed and $14.16 \%$ were depressed, however among subjects in the same age and calorie group but with low PAL 35.80\% were notdepressed and $64.20 \%$ were depressed. The percent population of not-depressed young subjects on CE diet with low PAL was significantly decreased (58.29\%; $\mathrm{p}<0.001$ ) and that of depressed population was significantly increased $(353.39 \% ; \mathrm{p}<0.001)$ compared to young subjects on CE diet and with high PAL. Results [Figure 4 (b)] reveals that of out 23 aged subjects on CE diet and with high PAL $86.96 \%$ and $13.04 \%$ were categorized as not-depressed and depressed respectively. Again out of 48 aged subjects on CE diet but with low PAL the not-depressed and depressed populations were found to be $29.17 \%$ and $70.83 \%$ respectively [Figure 4 (b)]. Results also reveals that among aged consuming CE diet, low PAL significantly decreased $(66.45 \%, \mathrm{p}<0.001)$ not-depressed population while significantly increased $(443.17 \%$; $p<0.001)$ the depressed population compared to population of the same age and calorie group but with high PAL. No significant differences ( $p>0.05$ ) in not-depressed and depressed populations were noted between young and aged subjects consuming CE diet and with high PAL [Figure 4 (b)]. Similarly CE diet consuming aged subjects with low PAL showed no significant change ( $p>0.05)$ in not-depressed and depressed populations [Figure 4(a) and (b)] compared to their young counterparts. CE diet consuming young subjects with low PAL showed significant decrease $(58.83 \%$; $<<0.001)$ in not-depressed and significant increase (392.33\%; $\mathrm{p}<0.001)$ in depressed populations [Figure 4 (a) and (b)] when compared with aged subjects in the same calorie group but with high PAL. Conversely, when aged subjects on CE diet and with low PAL were compared to young subjects in the same calorie group but with high PAL, the aged subjects showed significantly lower $(66.02 \%$; $<<0.001)$ not-depressed populations while depressed populations were significantly increased (400.21\%; $\mathrm{p}<0.001)$. 

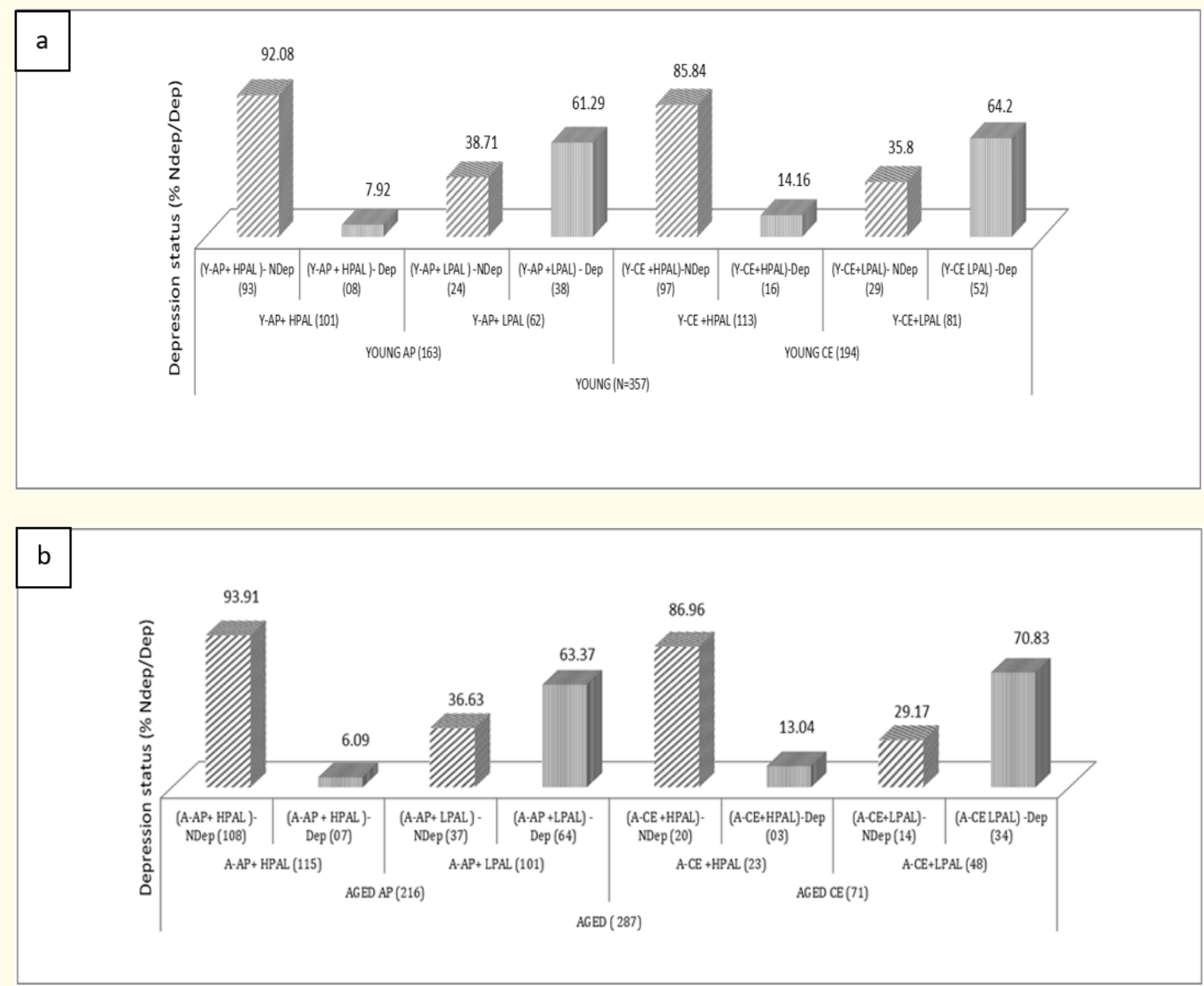

Number within parenthesis represents number of (a) Young subjects and (b) Aged subjects in respective groups. Y represents Young; A represents Aged; AP indicates At Par, CE indicates Calorie Excess ( $>30 \%$ of calorie requirement); HPAL indicates High PAL, LPAL indicates Low PAL and NDep represents not-depressed, Dep represents depressed.

Figure 4: Effect of PAL on CE-induced changes in Depression of (a) Young and (b) Aged subjects.

Results of logistic regression analysis (Table 2) reveals that excess calorie intake and aging had no significant effect on the outcome of occurrence of depression, however PAL affected the outcome of occurrence of depression in a negative manner (i.e., number of depressed subjects decreased with high PAL). In addition, PAL was identified as a stronger predictor of depression. In the present models chi square had 3 degrees of freedom, a value of 216.747 and $\mathrm{p}<0.001$ for depression (Table 2) indicating that the models had a poor fit, with the models containing only the constant suggesting that the predictors had a significant effect and created essentially a different model. Well-fitting models showed non-significance on the H-L goodness-of-fit test [38]. This desirable outcome of non-significance indicated that the model prediction did not significantly differ from the observed results (Table 2). In the present analysis H-L statistic had the significance of 0.307 for depression (Table 2) which indicated statistically not significant and therefore the present models were quite good fit. Nagelkerke's pseudo $\mathrm{R}^{2}$ value of 0.394 (Table 2) for depression in-

\begin{tabular}{|c|c|c|c|c|c|}
\hline \multirow{3}{*}{$\begin{array}{l}\begin{array}{l}\text { Independent } \\
\text { variables }\end{array} \\
\text { Risk factors }\end{array}$} & \multicolumn{5}{|c|}{ Dependent variable } \\
\hline & \multicolumn{5}{|c|}{ Depression } \\
\hline & B & SE & OR & CI & $\begin{array}{c}\mathrm{p} \\
\text { value }\end{array}$ \\
\hline Age & -0.002 & 0.007 & 0.998 & $0.983,1.012$ & $>0.05$ \\
\hline Calorie intake & 0.0001 & 0.0001 & 1.000 & $1.000,1.001$ & $>0.05$ \\
\hline PAL & -2.782 & 0.219 & 0.062 & $0.040,0.095$ & $<0.001$ \\
\hline $\begin{array}{l}\text { Nagelkerke's p } \\
\text { Model } X^{2}: 216 \\
\text { H \& L Test: } p= \\
N=644\end{array}$ & $\begin{array}{l}\text { seudo } \mathrm{R}^{2} \\
747, \mathrm{df}= \\
0.30\end{array}$ & $\begin{array}{l}0.394 \\
, p<0.00\end{array}$ & & & \\
\hline
\end{tabular}

Table 2: Logistic regression analysis of the effects of age, calorie intake and PAL on Depression of human male subjects.

B: Regression Coefficient; SE: Standard Error; OR: Odds Ratio; CI: Confidence Interval; PAL: Physical Activity Level; H \& L: Hosmer and Lemeshow; N: Study Population. 
dicated a weak relationship of $39.4 \%$ between predictors and prediction of depression. Age and calorie intake showed no significant effect on depression status; whereas, increase in PAL significantly decreased the likelihood of being depressed among both the young and aged population.

\section{Discussion}

The assumption that aging is associated with an increased risk of depression is a recurring theme in the gerontological literature. Yet empirical studies that have investigated the relation between aging and depression do not show consistent support for this assumption or hypothesis. Indeed, some investigators contend that the thrust of empirical evidence favors a view of elderly persons as relatively immune from depression, at least in contrast to their younger adult counterparts. Diverse measurement approaches, coupled with flaws in design and analysis, make it difficult to draw any definitive conclusions regarding the age-depression relation [40]. The question remains: why are not all elderly persons suffering from depression? The answer may lie in the interaction of environment, life stresses, and the internal adaptive capacities of the individual [41]. Applying a developmental perspective on biological change, psychological adaptation, and stress processes throughout adulthood shows that risk for depression onset in young adults is typified more through psychological vulnerability and stress, as well as genetic factors, while risk for depression in older adults typified more through comorbid medical and neurological disorder [42].

The present study includes subjects who belong to lower middle (III) and upper lower (IV) socio economic class [27,28]. Significantly $(p<0.05)$ higher percentage of carbohydrate content of food consumed by the entire study population compounded with deficiency of micronutrients faithfully validate their self- reported economic status. The study has also found that, significant $(\mathrm{p}<0.001)$ excess energy consumed by the young and aged subjects in the CE category compared to their AP counterparts, accounts for the difference in total carbohydrate intake. Reports on correlation between excess calorie consumption and depression are scanty. However, association of obesity with depression has been reported in a number of earlier studies [43-45].

In the present study significant decrease $(\mathrm{p}<0.05)$ in the percent population of excess calorie consuming not-depressed aged subjects compared to their young counterparts and significant increase in the percent population of depressed aged subjects on CE diet with respect to the young CE subjects (Figure 3) suggest that excess calorie intake in aged probably induces depressive symptoms. Though the exact mechanism of induction of depression by excess calorie consumption in the aged is not known, explanation of the effect is rather an extrapolation of various mechanisms described in available literature [46]. In this context the effect of calorie restriction on depression is of utmost importance which may help to explain the relation of depression with intake of excess calorie. Available reports describing relation between obesity and depression state that obesity is the consequence of sustained positive energy balance over time [47]. The factors that influence energy balance can be considered as relating to the host (i.e., people), the environment (the set of external factors to which people are exposed) and the vector (food and drink). These factors interact in a complex way to influence eating and drinking patterns as well as behavioral activity [48].

People affected by obesity are often self-conscious about their appearance or their physical abilities. They withdraw or are excluded from social activities. They find themselves feeling more isolated from friends, co-workers and loved ones. All the while, their feelings of self-worth continue to fall. Similarly, depression is debilitating, and someone suffering from it may be less motivated to exercise, less able to follow a diet closely, less able to take the time to prepare healthy foods versus grabbing something fast, and less convinced that taking care of their health is a good use of time. All these feelings develop from Stigma and discrimination toward obese leading to numerous consequences for their psychological and physical health [49].

The root cause of expression of depressive behavior by both the young and the aged participants of the present study is difficult to reveal. Though all the subjects identified to be deficient in proteins, essential vitamins, minerals and to some extent lipids, still all of them are not equally affected from depression. This is because factors that influence nutrition can be considered as relating to the host (i.e., people), the environment (the set of external factors to which people are exposed) and the vector (food and drink) [48]. Moreover very low quantity of essential amino acids in the diet of the study population is more likely. Lack of balanced diet with associated poor absorption due to aging may precipitate depression. The essential amino acids tyrosine and tryptophan are the raw ingredients for synthesis of dopamine and serotonin [50,51] and low levels of these two neurotransmitters are linked with depression. According to Marcus and Berry [52] depression in the aged may relate to physiologic changes associated with aging, such as dementia. They also find medical, social, and environmental issues as causative factors.

Physical inactivity is a modifiable risk factor for various life style diseases including depression [53]. Adults with Major Depressive Disorder (MDD) engage in low levels of physical activity (PA) and high levels of sedentary behavior (SB). PA and SB are independent predictors of mortality [54]. Controversy continues if depression leads to over eating (Binge eating) or the reverse. 
Results of the present study [Figure 4(a) and (b)] also show that high PAL compared to low PAL decreases depressed population and increases not depressed population irrespective of age and calorie intake. In this context an interesting study report on Indian population with 14227 male and female subjects [55] reveals that the prevalence of inactivity ( $54.4 \%$ males) is higher than that of all other modifiable risk factors. Routine Moderate physical activity level is associated with improved psychological well-being probably through reduced stress, anxiety and depression [56].

The present findings of goodness-of-fit test (Table 2) may further suggest by reconfirming using Pearson correlation statistical analysis [57] which determines the association of independent variable with the dependent variable (depression) (a) a correlation between depression and (i) aging $r(642)=0.047, p>0.05$; (ii) calorie intake $r(642)=-0.078 p<0.05$; and (iii) PAL $r(642)=0.562$ $\mathrm{p}<0.001$ (Table 3), (b) association of depression with aging (0.047) and calorie intake (-0.078) are weak and that with PAL (0.562) is moderate (Table 3) and (c) association between depression and aging or PAL is positive but with calorie intake the association is negative.

\begin{tabular}{|l|c|c|}
\hline \multirow{2}{*}{ Independent Variable } & \multicolumn{2}{|c|}{ Dependent Variable } \\
\cline { 2 - 3 } & \multicolumn{2}{|c|}{ Depression } \\
\cline { 2 - 3 } & Pearson Correlation & p value \\
\hline Age & 0.047 & $>0.05$ \\
\hline Calorie intake & -0.078 & $<0.05$ \\
\hline PAL & 0.562 & $<0.001$ \\
\hline
\end{tabular}

Table 3: Study of the direction and strength of association between Independent and dependent variables using Pearson correlation.

While the importance of lifestyle factors such as diet and physical activity level are generally acknowledged in the research literature on depression, the mechanisms of their potential influence are often not fully appreciated. Sleep, not taken as a parameter in the present study, can also influence several physiological pathways associated with depression. A bi-directional relationship likely exists between depression and these lifestyle factors, thereby creating a potentially increasing cycle of influence.

The importance of these lifestyle factors has been highlighted in a recent paper by Jacka., et al. [58] who argued that depression should be included under the umbrella of non-communicable diseases influenced by lifestyle factors, with increasing efforts directed toward prevention through the promotion of lifestyle changes. While these lifestyle factors are significant in the etiology and maintenance of depression, a multitude of other lifestyle influences may also be important. These include chronic stress, social influences, mental and physical effects associated with medical diseases and even chronic pain. It is these influences, plus a large array of psychological, genetic and biological factors that often make the treatment of depression difficult. The present study is believed to add to the existing knowledge on mental health problems of people in a part of eastern India with micronutrient deficiency that belong to lower socioeconomic class and the outcome of intervention of variable calorie intake and physical activity. Finally a limitation associated with the present study is that significant portions are correlational and/or epidemiological, thereby limiting conclusions about causation.

\section{Conclusion}

It may be concluded from the present study (Figure 5) that (1) Consumption of CE diet by the aged, unlike AP calorie diet, increases the percent population of depressed subjects; (2) Young subjects do not exhibit any significant difference in depressive outcome when on CE diet as compared to AP calorie consumption; (3) High PAL, unlike low PAL, masks the excess calorie-induced depressive symptoms among aged subjects.

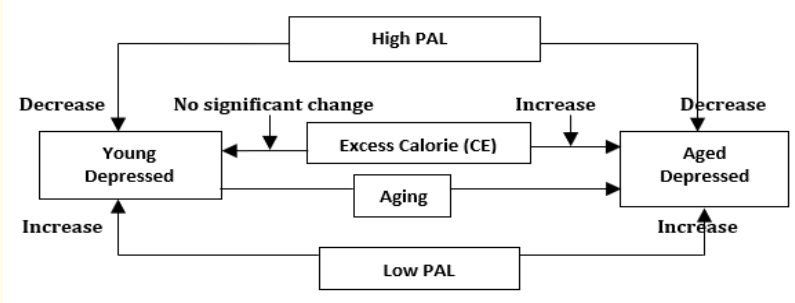

Figure 5: Schematic presentation of the influence of aging, excess calorie intake, and physical activity on depression.

\section{Acknowledgement}

This study was supported, in part, by the Minor Research Project [F.No.PSW-085/2014-2015 (ERO)] of Dr. Samir Kumar Ghosh, funded by the University Grants Commission, New Delhi. The authors express their indebtedness to Prof. S. Sengupta, Department of Statistics, University of Calcutta for his technical advice. The authors are also grateful to the Indian Council of Medical Research (ICMR), New Delhi, India, and Department of Biochemistry, University of Calcutta, Kolkata, India.

\section{Conflict of Interest}

No financial interest or any conflict of interest exists.

\section{Bibliography}

1. National Sample Survey Organization. Annual Report, (2011); World population prospects, (2012).

2. World population prospects: revision. United Nations: Department of Economics and Social Affairs; Population Estimates and Projections Section, Population Division, (2012). 
3. Amarya S., et al. "Changes during aging and their association with malnutrition". Journal of Clinical Gerontology and Geriatrics 6.3 (2015): 78-84.

4. Ory MG and Cox DM. "Forging ahead: linking health and behavior to improve quality of life in older people". Social Indicators Research 33.1-3 (1994): 89-120.

5. Rolls BA, Drewnowski A, Diet and nutrition. Birren JE, ed. Encyclopedia of Gerontology, Academic Press, San Diego, CA. 1 (1996): 429-440.

6. Omenn GS., et al. "Evidence of modifiable risk factors in older adults as a basis for health promotion and disease prevention programs". Hickey T, Speers MA, Prohaska TR, ed. Public Health and Aging (1997): 107-127. Johns Hopkins University Press, Baltimore.

7. Jackson TM. "Nutrition research and the elderly". Nutrition Reviews 52 (1994): S1-S53.

8. Moinpour CM., et al. "Quality of life end points in cancer clinical trials: review and recommendations". Journal of the $\mathrm{Na}$ tional Cancer Institute 81.7 (1989): 485-495.

9. Ware JE. "Standards for validating health measures: definition and content". Journal of Chronic Diseases 40.6 (1987): 473-480.

10. Stewart A and Hays RD. "Conceptual, measurement, and analytical issues in assessing health status of older populations. Hickey T, Speers MA, Prohaska TR, ed. Public Health and Aging (1997):163-189. Johns Hopkins University Press, Baltimore.

11. Power M., et al. "WHO Quality of Life Group. The World Health Organization WHOQOL-100: tests of the universality of quality of life in 15 different cultural groups worldwide". Health Psychology 18.5 (1999): 495-505.

12. Diener E. "Subjective well-being". American Psychologist 55.1 (2000): 34-43.

13. Harvey AG. "Sleep and circadian rhythms in bipolar disorder: Seeking synchrony, harmony, and regulation". American Journal of Psychiatry 165.7 (2008): 820-829.

14. Rao TSS., et al. "Understanding nutrition, depression and mental illnesses". Indian Journal of Psychiatry 50.2 (2008): 77-82.

15. Balsamo M., et al. "Cognitive Vulnerabilities and Depression in Young Adults". An ROC Curves Analysis: Depression Research and Treatment (2013); 2013 Article ID 407602, 8.

16. Biderman A., et al. "Depression and falls among community dwelling elderly people: a search for common risk factors". Journal of Epidemiology and Community Health 56.8 (2002): 631-636.
17. Lopresti AL., et al. "A review of lifestyle factors that contribute to important pathways associated with major depression: Diet, sleep and exercise". Journal of Affective disorders 148.1 (2013): 12-27.

18. Abildgaard A., et al. "A high-fat diet exacerbates depressive like behavior in the Flinders Sensitive Line (FSL) rat, a genetic model of depression". Psychoneuroendocrinology 36.5 (2011): $623-633$.

19. Camacho TC., et al. "Physical Activity and Depression: Evidence from the Alameda County Study". American Journal of Epidemiology 134.2 (1991): 220-231.

20. Hill JO., et al. "Energy Balance and Obesity". Circulation 126.1 (2012): 126-132.

21. Luppino F., et al. "Overweight, obesity and depression: a systematic review and meta-analysis of longitudinal studies". Archives of General Psychiatry 67.3 (2010): 220-229.

22. Wells JL and Dumbrell AC. "Nutrition and ageing: assessment and treatment of comprised nutritional status in frail elderly patients". Clinical interventions in aging 1.1 (2006): 67-79.

23. Gariballa SE. "Nutritional support in elderly patients". The Journal of Nutrition Health and Aging 4.1 (2000): 25-27.

24. Whitehead C and Finucane P. "Malnutrition in elderly people". Australian and New Zealand Journal of Medicine 27.1 (1997): $68-74$

25. Schuch FB., et al. "Exercise improves physical and psychological quality of life in people with depression: A meta-analysis including the evaluation of control group response". Psychiatry Research 241 (2016): 47-54.

26. Kruisdijk FR., et al. "Effect of running therapy on depression (EFFORT-D). Design of a randomized controlled trial in adult patients [ISRCTN 1894]". BMC Public Health 12 (2012): 50.

27. Kumar N., et al. "Kuppuswamy's Socioeconomic Scale: Updating Income Ranges for the Year 2012". Indian Journal of Public Health 56.1 (2012): 103-104.

28. Tabassum N and Rao RLL. "An updated Kuppuswamy's socioeconomic classification for 2017". International Journal of Health Sciences and Research 7.5 (2017): 365-367.

29. Gibson RS and Ferguson EL. An interactive 24-hr recall for assessing the adequacy of iron and zinc intakes in developing countries, ILSI Press, Washington DC. (1999).

30. Yunsheng MA., et al. "Number of 24-Hour Diet Recalls Needed to Estimate Energy Intake". Annals of Epidemiology 19.8 (2009): 553-559.

31. FAO. Dietary Assessment: A resource guide to method selection and application in low resource settings. Rome (2018). 
32. Ramey Dr., et al. "in Spilker Quality of Life and Pharmacoleconomics in Clinical Trials, 2nd ed, The Health Assessment Questionnaire 1995- Status and Review". Philadelphia: Lippincott-Raven Pub., (1995): 227-237.

33. Radloff LS. "The CES-D scale: A self-report depression scale for research in the general population". Applied Psychological Measurement 1 (1977): 385-401.

34. Bharathi AV., et al. "The development and characteristics of a physical activity questionnaire for epidemiologic studies in urban middleclass Indians". Indian Journal of Medical Research 111 (2000): 95-102.

35. FAO/WHO/UNU Expert Consultation Report on Human energy requirements: Principles and Definitions. Retrieved 2009-10-15 (2004).

36. Yang-chun F., et al. "The application of Student's t-test in internal quality control of clinical laboratory". Frontiers in Laboratory Medicine 1.3 (2017): 125-128.

37. Santos e Silva F and Vieira de Azevedo CA. "Comparison of means of agricultural experimentation data through different tests using the software Assistat". African Journal of Agricultural Research 11.37 (2016): 3527-3531.

38. Bursac Z., et al. "Purposeful selection of variables in logistic regression". Source Code for Biology and Medicine 3 (2008): 17.

39. Dietary Reference Intakes for Calcium, Phosphorous, Magnesium, Vitamin D, and Fluoride (1997); Dietary Reference Intakes for Thiamin, Riboflavin, Niacin, Vitamin B6, Folate, Vitamin B12, Pantothenic Acid, Biotin, and Choline (1998); Dietary Reference Intakes for Vitamin C, Vitamin E, Selenium, and Carotenoids (2000); Dietary Reference Intakes for Vitamin A, Vitamin K, Arsenic, Boron, Chromium, Copper, Iodine, Iron, Manganese, Molybdenum, Nickel, Silicon, Vanadium, and Zinc (2001); Dietary Reference Intakes for Energy, Carbohydrate, Fiber, Fat, Fatty Acids, Cholesterol, Protein, and Amino Acids (2002/2005); and Dietary Reference Intakes for Calcium and Vitamin D (2011). These reports may be accessed via www.nap.edu.

40. Newmann JP. "Aging and depression". Psychology and Aging 4.2 (1989): 150-165.

41. Jarvik LF. "Aging and Depression: Some Unanswered Questions". Journal of Gerontology 31.3 (1976): 324-326.

42. Karel MJ. "Aging and depression: Vulnerability and stress across adulthood". Clinical Psychology Review 17.8 (1997): 847-879.

43. Jorm AF., et al. "Association of Obesity with Anxiety, Depression and Emotional Well-Being: A Community Survey". Australian And New Zealand Journal of Public Health 27.4 (2003): 434-440.

44. Atlantis E and Baker M. "Obesity effects on depression: systematic review of epidemiological studies". International Journal of Obesity 32.6 (2008): 881-891.
45. McLaren L., et al. "The relationship between body mass index and mental health". Social Psychiatry and Psychiatric Epidemiology 43.1 (2008): 63-71.

46. Al-Rasheed R., et al. "Malnutrition in elderly and its relation to depression". Int J Community Med Public Health 5.6 (2018): 2156-2160.

47. Hill JO and Commerford R. "Exercise, fat balance and energy balance". International Journal of Sports Nutrition 6.2 (1996): 80-92.

48. Romieu I., et al. "Energy balance and obesity: what are the main drivers?". Cancer Causes Control 28.3 (2017): 247-258.

49. Puhl RM and Heuer CA. "Obesity Stigma: Important Considerations for Public Health". American Public Health Association 100.6 (2010): 1019-1028.

50. Diehl DJ and Gershon S. "The role of dopamine in mood disorders". Comprehensive Psychiatry 33.2 (1992): 115-120.

51. Stockmeier CA. "Neurobiology of serotonin in depression and suicide". Annals of the New York Academy of Sciences 836 (1997): 220-232.

52. Marcus EL and Berry EM. "Refusal to eat in the elderly". Nutrition Reviews 56.6 (1998): 163-171.

53. Morgan W. Physical Activity, Fitness and Depression. In C.Bouchard, R.J. Shephard and T. Stephens (Eds.), Physical Activity, Fitness and Health Champaign, IL; Human Kinetics (1994): 796 - 813.

54. Schuch F., et al. "Physical activity and sedentary behavior in people with major depressive disorder: A systematic review and meta-analysis". Journal of Affective Disorders 210 (2017):139-150.

55. Anjana RM., et al. "Physical activity and inactivity patterns in India - results from the ICMR - INDIAB study (Phase- 1) [ICMR-INDIAB-5]". International Journal of Behavioral Nutrition and Physical Activity 11.1 (2014): 26.

56. Dunn AL., et al. "Physical activity dose-response effects on outcomes of depression and anxiety". Medicine and Science in Sports and Exercise 33.6 (2001): S587-S597.

57. Mukaka MM. "A guide to appropriate use of Correlation coefficient in medical research". Malawi medical journal 24.3 (2012): 69-71.

58. Jacka FN., et al. "Moving towards a population health approach to the primary prevention of common mental disorders". BMC Medicine 10 (2012): 149.

\section{Volume 3 Issue 8 August 2019}

(C) All rights are reserved by Mrinal K Poddar., et al. 